\title{
EL REALISMO DE LOS SUPUESTOS Y LA VERIFICACIÓN DE LAS LEYES ECONÓMICAS
}

\author{
MANuEl Gollás \\ El Colegio de México
}

\section{LOS ENFOQUES INSTRUMENTALISTA Y REALISTA}

EsTe TRABAjo constituye una exploración breve del problema que presenta la construcción de la teoría económica en relación con la naturaleza de sus proposiciones y supuestos. Se trata específicamente el problema del realismo de los supuestos y de la verificación de teorías. ${ }^{1}$

Las discusiones acerca de si la economía es o no una ciencia deductiva datan cuando menos desde J. S. Mill. Su punto de vista al respecto está expresado claramente en su afirmación de que "Es vano esperar que pueda llegarse a la verdad, en la economía política o en cualquiera otra rama de las ciencias sociales, mirando los hechos de lo concreto, con toda la complejidad de que la naturaleza los ha rodeado, y empeñarse en extraer una ley general mediante un proceso de inducción por la comparación de detalles; no queda otro método que el a priori, o que la especulación abstracta." 2

En la actualidad el mismo punto de vista ha sido expresado por Lionel Robbins, quien afirma que "...las proposiciones de la teoría económica, al igual que toda teoría científica, son obviamente deducciones de una serie de postulados. Y los principales postulados son aquellos supuestos que involucran de algún modo simple e indiscutible los hechos de la experiencia... Son hechos tan comunes de la vida diaria que solamente tienen que ser expresados para que se les reconozca como obvios". 3 La característica esencial de estos autores es que en su opinión los postulados

1 Una discusión amplia sobre la estructura y significado de teorías científicas puede encontrarse en E. Nagel, The Structure of Science, Nueva York, Harcourt, Brace and World, Inc. 1961, Cap. 5; N. Cambell, Foundation of Science, Cap. 6; R. B. Braithwaite, Scientific Explanation, Cambridge, Mass., Harper and Row Publishers, 1968; C. G. Hempel, "The Theoretician's Dilema", en H. Feigl et al., Minnesota Studies in the Philosophy of Science, Vol. II. En relación con los enfoques instrumentalistas $\mathrm{y}$ realistas en la filosofía de la ciencia pueden consultarse P. K. Feyerabend, "Realism and Instrumentalism", en M. Bunge (Comp.), The Critical Approach: Essays in Honor of Karl Poper, 1963; E. Nagel, The Structure of Science, Cap. 6; y R. Carnap, Foundations of Logic and Matematics, 1939.

2 John S. Mill, "On the Definition of Political Economy and on the Method of Investigation Proper to it" en Essays on Some Unsettled Question of Political Economy, Londres, 1844. Citado por Fritz Machlup en "The Problem of Verification in Economics", The Southern Economic Journal, julio de 1955, p. 6.

3 Lionel Robins, An Essay on the Nature and Significance of Economic Science, Londres, 1973, pp. 78 y 79. 
de la teoría económica no tienen que estar sujetos a prueba empírica alguna y que no solamente son verdaderos sino evidentes por sí mismos. Similarmente, otros economistas opinan que los supuestos de la teoría económica no necesitan sujetarse a prueba empírica alguna, “... . sino solamente los pronósticos que se deducen de ellos".4

Recientemente, Friedman ha adoptado un punto de vista semejante en relación con la naturaleza y el papel de los supuestos económicos. Friedman considera que los postulados económicos, al igual que el resto de los supuestos científicos, no tienen por qué ser realistas y que no necesitan sujetarse a pruebas empíricas. La selección de postulados no es una cuestión de verdad o de validez empírica sino algo que debe decidirse refiriéndose a la utilidad de la teoría. Para Friedman “...la pregunta relevante en relación a los 'supuestos' de una teoría no consiste en si éstos son descriptivamente 'realistas' ya que nunca lo son, sino en si constituyen una aproximación suficientemente buena para el propósito en mente. Y esta cuestión puede responderse únicamente al ver si se obtienen predicciones de exactitud suficiente". ${ }^{5}$ Esto significa que al hablar de los postulados de la teoría económica debe abandonarse el concepto de verdad.

El enfoque adoptado por los autores anteriores puede identificarse con el punto de vista instrumentalista de las teorías de la economía.

En el extremo opuesto se encuentran los economistas que insisten en la verificación independiente de los supuestos de la economía. Así, Hutchison demanda la prueba de todos los supuestos económicos centrales. ${ }^{6}$ Considera que una teoría más realista necesariamente implicará supuestos más realistas. ${ }^{7}$ Para estos empiricistas ${ }^{8}$ "el sistema deductivo hipotético construido sobre supuestos irreales o no verificables, está condenado ya sea por descriptivo o por carecer de contenido empírico alguno, sin significado predictivo o explicativo, sin aplicación a problemas o a datos del mundo real. ${ }^{9}$ Para Hutchison " . . las proposiciones de la teoría pura no tienen en sí mismas valor pragmático o 'significado causal" ".10 Por lo tanto, esta postura demanda supuestos realistas y empíricamente verificables en la teoría económica. Este enfoque puede identificarse con el punto de vista realista de las teorías.

Debe señalarse que en ambas posiciones, la instrumentalista y la realista, el concepto de "realismo" y el de "verificabilidad" de los supuestos

4 Fritz Machlup, "The Problem of Verification in Economics", Southern Economic Journal, julio de 1955, p. 7.

5 Milton Friedman, Essays in Positive Economics, Chicago, University of Chicago Press, 1953, p. 15.

6W. G. Hutchison, The Significance and Basic Postulates of Economic Theory, Londres, 1973 , p. 78 y 79 .

7 Op. cit., p. 120.

8 Machlup toma a Hutchison como miembro del grupo ultraempiricista, pero esto no parece correcto, ya que Hutchison dice explícitamente: "Las proposiciones deben ser susceptibles de verificación empírica o reducibles a proposiciones verificables mediante la deducción lógica o matemática... Es decir, no precisan ser realmente sometidas a prueba o inclusive susceptibles prácticamente de verificación en las condiciones técnicas presentes o futuras... ni tiene sentido alguno hablar de una especie de prueba 'absoluta' que determinará definitivamente si una proposición es 'absolutamente' verdadera o falsa", op. cit., p. 9.

9 F. Machlup, op. cit., p. 8.

10 Hutchison, op. cit., p. 162. 
están estrechamente identificados ya que con frecuencia por "realismo" se quiere significar "verificabilidad" y viceversa.

\section{LAS PROPOSICIONES DE UNA TEORÍA}

Veamos ahora más de cerca los argumentos para apoyar la necesidad de postulados "realistas" o "no realistas" en la economía. En la construcción del conocimiento económico se acepta generalmente que existen dos tipos diferentes de postulados: proposiciones sin contenido empírico y proposiciones con contenido empírico. ${ }^{11}$ Se hará ahora una explicación de estos dos argumentos dentro del contexto de la controversia "instrumentalista" versus "realista".

Las proposiciones sin contenido empírico. Estas proposiciones han recibido nombres diferentes, pero su característica común es que son conceptos abstractos que además de carecer de significado empírico, no lo necesitan para ser útiles en la construcción de la teoría económica.

Algunos economistas ${ }^{12}$ se refieren a estas proposiciones como hipótesis "fundamentales" (heurísticas) o "hipótesis de alto nivel" o "generalizaciones de alto nivel". ${ }^{13}$ Ejemplos de generalizaciones de alto nivel o supuestos fundamentales en la teoría económica son "los individuos actúan racionalmente"; "tratan de aprovechar mejor sus oportunidades"; "los empresarios prefieren mayores utilidades ante riesgos iguales". ${ }^{14}$

Ysander hace una distinción semejante de los postulados. ${ }^{15}$ En su opinión, la economía utiliza conceptos abstractos, subjetivos e ideales en sus postulados. Estos conceptos "deben tratarse como variables teóricas que tienen una interpretación sólo parcialmente empírica". ${ }^{16}$ Ysander llama a estos conceptos "términos no empíricos". Desempeñan una función "como instrumento de la construcción de la teoría más que como símbolos de objetos concretos". ${ }^{17}$ Ejemplo de términos no empíricos es lo que los soció-

11 La discusión sobre la naturaleza y función de los términos y proposiciones de una teoría no se limita a la teoría económica. Una discusión amplia sobre este tema puede encontrarse en: F. Achinstein "On the Meaning of Scientific Terms" en Journal of Philosophy, 1964; E. Maxwell, "The Ontological Status of Theoretical Entities", en H. Feigl (Comp.), Minnesota Studies in the Philosophy of Science, Vol. III; P. K. Feyerabend, "On the Meaning of Scientific Terms", en Journal of Philosophy, 1965; W. V. O. Quine, From a Logical Point of View, "Two Dogmas of Empiricism".

12 F. Machlup, op. cit., pp. 8-11.

13 F. Machlup cita a R. B. Braithwaite para apoyar su argumento: "A medida que se eleva la jerarquía de las hipótesis de generalidad creciente, los conceptos involucrados en estas hipótesis dejan de ser propiedades de los objetos directamente observables y se convierten en su lugar en conceptos "teóricos" -átomos, electrones, campos de fuerza, procesos mentales inconscientes- que están conectados con los hechos observables por relaciones lógicas complicadas”. R. B. Braithwaite, Scientific Explanation, Cambridge, 1953, p. ix.

14 F. Machlup, op. cit., p. 10.

15 Bengt-Christer Ysander, "Truth and Meaning of Economic Postulates" en Hugo Hegeland (Comp.), Money, Growth and Methodology, Suecia, 1961.

16 Ibid., p. 333.

17 Ibid., p. 328. 
logos llaman "variables inferidas". Las caracteriza el hecho de que "no pueden identificarse fácilmente con ninguna propiedad empírica de la conducta humana". ${ }^{18}$ Ysander simpatiza con lo que llama enfoque pragmático, representado por M. Friedman, a quien considera ". . . un ejemplo maduro de pragmatismo radical". ${ }^{19}$

La existencia de conceptos abstractos y empíricamente vacíos en la teoría económica ha sido señalada también por Hutchison, quien llama a este tipo de afirmaciones "proposiciones de teoría pura". ${ }^{20}$ Ejemplos de estas proposiciones son: "bajo competencia perfecta, las empresas son de tamaño óptimo". Hutchison considera que las "proposiciones de teoría pura" son definiciones analítico-tautológicas que juegan un papel importante en la construcción teórica. La teoría económica ha sido construida de este modo y por ello a menudo ha sido acusada de ser circular y tautológica.

Desde este punto de vista, las proposiciones de teoría pura son empíricamente vacías ya que son "incondicionalmente verdaderas y puesto que no son confirmables ni contradecibles, no pueden decirnos nada nuevo acerca del mundo". Sin embargo, son útiles en la investigación económica ya que "llaman la atención sobre las implicaciones de nuestras definiciones... y revelan relaciones inesperadas entre ellas". ${ }^{21}$

Entonces, aparentemente no parece haber mucho desacuerdo entre el punto de vista de los instrumentalistas (Friedman, Ysander, etc.) y los realistas (Hutchison, y otros) en relación con la necesidad de proposiciones sin contenido empírico en la construcción de teorías. A este respecto no se encuentra conflicto alguno entre ambos puntos de vista. Como se verá, el desacuerdo surge del hecho de que para los realistas solamente algunos de los supuestos de la teoría (las proposiciones de teoría pura) no requieren contenido empírico ni ser verificables. Por otra parte, para los instrumentalistas extremos, todos los supuestos de una teoría no solamente carecen de contenido empírico ${ }^{22}$ sino que tampoco lo necesitan. Gran parte del conflicto entre instrumentalistas y realistas surge debido a que ninguna de las dos partes distingue las implicaciones empíricas y el papel de los distintos tipos de proposiciones que constituyen los supuestos de una teoría.

Proposiciones con contenido empírico. Los supuestos de este tipo han sido llamados "supuestos específicos" que se supone corresponden a hechos o condiciones observadas. ${ }^{23}$ Ejemplos de este tipo de proposiciones en la teoría económica son del tipo: "el gasto en sal de mesa es una porción muy pequeña en el presupuesto de la mayoría de los hogares". Estos supuestos han sido llamados también "proposiciones de la teoría aplicada" y corresponden a afirmaciones del tipo " $p$ por lo tanto $q$ ". En esta afirmación, la premisa $p$ se considera verdadera empíricamente. Ejemplos

18 Ibid., p. 331.

19 Ibid., p. 330.

20 W. G. Hutchison, op. cit., p. 24.

21 Ibid., p. 30.

22 Esta posición llevada al extremo es absurda ya que implica que no se podria saber a cuál fenómeno se refiere la teoría.

23 F. Machlup, op. cit., p. 8-9. 
de proposiciones de teoría aplicada en la economía son: el mercado $X$ opera en condiciones de competencia perfecta, por lo tanto, el tamaño de las empresas es óptimo. $\mathrm{O}$ bien, $M$ ha aumentado y $V$ y $Y$ han permanecido constantes, por lo tanto $P$ se ha elevado. ${ }^{24}$

Se han señalado antes las diferencias entre proposiciones con y sin contenido empírico dentro del contexto de los puntos de vista realista e instrumentalista de las teorías, y se han tomado las posiciones extremas para aclarar el significado de estos términos. Sin embargo, muchos economistas cómodamente toman la posición intermedia en esta cuestión.

\section{EL REALISMO Y LA VERIFICACIÓN DE LOS SUPUESTOS EN LA ECONOMÍA}

Volvemos ahora al problema de la verificación de los supuestos de la teoría económica en el contexto de la controversia entre realismo e instrumentalismo.

Respecto de las proposiciones sin contenido empírico, ni instrumentalistas ni realistas exigen la verificación. Para el realista no tiene significado pedir tal verificación ya que estas proposiciones son necesariamente verdaderas, son tautológicas. Por su parte, los instrumentalistas consideran innecesaria, sin importancia ni significado la verificación de proposiciones sin contenido empírico (es decir, aquel tipo de proposiciones que según esta corriente constituyen una teoría) ya que no se refieren a cosas concretas sino que son sólo instrumentos de la construcción de teorías e inferencia.

El problema de verificación de proposiciones con contenido empírico es pertinente al enfoque realista. Verificar significa aquí encontrar si una proposición es verdadera o falsa en un sentido empírico. Por otra parte, la verificación de proposiciones con contenido empírico desde el punto de vista instrumentalista no tiene sentido, ya que éstas no constituyen parte de la estructura formal de los supuestos básicos de una teoría.

Ejemplos del enfoque realista son los que adoptan economistas como Hutchison quienes reconocen la existencia de proposiciones no realistas (proposiciones de teoría pura), pero recomiendan insistentemente la verificación de todos los supuestos económicos centrales. Piden más "supuestos realistas" para tener una teoría "más realista". ${ }^{25}$ Algunos economistas que tienen una posición menos enfática respecto a la verificabilidad de los supuestos en economía, admiten la posibilidad de que algunos supuestos no requieren de prueba empírica independiente alguna. ${ }^{26}$ Lo que debe hacerse en un sistema deductivo como el de la economía es probar las hipótesis de nivel más bajo. ${ }^{27}$

En contraste al enfoque realista anterior tenemos a instrumentalistas como Ysander quien afirma que "... en lugar de olvidar simple-

24 Este último ejemplo se refiere a la teoria cuantitativa del dinero que establece que $M V=P Y$, en donde $M=$ cantidad de dinero en circulación, $V=$ velocidad del dinero; $Y=$ nivel del ingreso nacional y; $P=$ nivel de precios.

$25 \mathrm{~W}$. Hutchison, op. cit., p. 83.

26 F. Machlup, op. cit., p. 10-11.

27 Ibid., p. 11. 
mente la cuestión de verdad o falsedad y admitir abiertamente falsedades en los postulados, tal como hacen los pragmatistas, los postulados pueden analizarse como algo que contiene variables teóricas que los hace inaccesibles a la prueba directa y ciertamente quitan todo sentido al problema de la validez. ${ }^{28}$

Según se ha visto, la controversia entre los enfoques instrumentalista y realista en la teorización económica gira alrededor de la necesidad de tener supuestos realistas. El concepto de "realismo de los supuestos" aparece en estrecha asociación con conceptos como "verificabilidad", "proposiciones con contenido empírico", "proposiciones sin contenido empírico", y "verdad o falsedad".

El uso y la interpretación de todos estos términos dan cuenta de las principales diferencias entre los enfoques instrumentalista y realista. En la sección siguiente se intenta explorar las condiciones bajo las cuales es importante establecer supuestos realistas en la construcción de la teoría económica.

\section{TEORÍAS Y MODELOS}

Papandreou ${ }^{29}$ ha elaborado un ejemplo concreto de cómo se construyen y confirman (verifican) la teoría y los modelos en la economía, aunque sin relacionar sus argumentos con la controversia descrita en párrafos previos. Después de delinear la tesis de este autor, las relacionaremos con el enfoque realista $v s$ el instrumentalista.

El argumento de Papandreou es el siguiente: Supóngase un sistema de ecuaciones que describen ciertas relaciones macroeconómicas.

$$
\begin{aligned}
& Y=C+I+G \\
& Z=Y-T \\
& C=a+b Z \\
& I=u+v Y \\
& T=r Y
\end{aligned}
$$

El sistema consiste de seis variables $(Y, C, I, G, Z$ y $T)$ y cinco parámetros estructurales $(a, b, u, v, \mathrm{y} r) ; Y$ es el valor del ingreso nacional, $C$ el gasto global en consumo, $I$ el gasto global de inversión, $G$ el gasto público total, $T$ el total de, impuestos al ingreso recibidos por el gobierno, y $Z$ es el ingreso disponible.

Una relación es un conjunto de valores de $Y, C, I, G, Z$ y $T$ que satisfacen nuestro sistema de ecuaciones. La idea puede expresarse de la manera siguiente:

$$
\begin{aligned}
& F_{1}=\{(Y, I, C, Z, T, G) \mid Y=C+I+G\} \\
& F_{2}=\{(Y, I, C, Z, T, G) \mid Z=Y-T\}
\end{aligned}
$$

29 Andreas G. Papandreu, Economics as a Science, Nueva York, 1958. 


$$
\begin{aligned}
& F_{3}=\{(Y, I, C, Z, T, G) \mid C=G+b Z\} \\
& F_{4}=\{(Y, I, C, Z, T, G) \mid I=u+v Y\} \\
& F_{5}=\{(Y, I, C, Z, T, G) \mid T=r Y\}
\end{aligned}
$$

Si reemplazamos $x$ por $(Y, I, C, Z, T, G)$ tendremos:

$$
\begin{aligned}
& x \varepsilon F_{1} \\
& x \varepsilon F_{2} \\
& x \varepsilon F_{3} \\
& x \varepsilon F_{4} \\
& x \varepsilon F_{5}
\end{aligned}
$$

en donde $\varepsilon$ quiere decir "es un elemento de" y $x$ es un vector en un espacio vectorial $R^{6}$.

La expresión anterior constituye un ejemplo de proposición abierta (función proposicional) porque para algunos valores de $x$ la proposición es falsa y verdadera para otros.

Lo que hace el economista es observar los valores de $Y, I, C, Z, T$ y $G$ (es decir, de $x$ ) de acuerdo con alguna regla $E$ de observación que satisface las relaciones $F_{i}(i=1, \ldots, 5)$. Supóngase que existen $\alpha$ actos de observación según la regla $E$. Los economistas construyen sistemas deductivos de la forma siguiente:

$$
\underset{a}{\forall} \underset{x}{\forall}\left[(\alpha, x) \varepsilon E \Rightarrow x \varepsilon F_{i}\right]
$$

En palabras: Para todos los actos de observación $\alpha$ de $x$, si el acto de observación se realiza de acuerdo con la regla $E$, es decir, si las parejas $(\alpha, x)$ son elementos de $E$, entonces estos valores observados serán elementos de $F_{1}, F_{2}, \ldots, F_{5}$ (satisfarán las relaciones del modelo).

Las proposiciones del tipo 1 son de carácter universal y tienen con-, tenido empírico; por lo tanto un solo caso contradictorio bastaría para hacerlas falsas. Los economistas encontrarán entonces muchos casos en que las proposiciones del tipo 1 son falsas. Sin embargo, al encarar esta situación, los economistas a menudo arguyen que el sistema fue ideado para cierto tipo de economía o para cierto período histórico y que por ello el sistema no da predicciones correctas. Si aceptamos restricciones de este tipo, entonces 1 no es una teoría. Si 1 ha de ser una teoría tenemos que aceptar las predicciones de 1 sin restricciones.

Una situación alternativa es la de dar a 1 una especificación del contexto de su aplicabilidad en términos generales. En lugar del término contexto se utilizará la expresión espacio social. El espacio social debe ser caracterizado independientemente de la información básica contenida en los postulados, es decir, no debe caracterizarse el espacio social dando a las ecuaciones una cierta forma. Hecha esta aclaración, denotemos por $k$ los actos de observación dirigidos a identificar el espacio social, y $K^{r}$ la clase de todos los actos de observación cuyo resultado es el espacio social $r$. Los actos de observación deben realizarse conjuntamente, pero independientemente de los actos de observación $\alpha$ en $x$. Esto se expresará mediante $\alpha P_{k}$. 
La proposición anterior (1) adquiere ahora la forma

$$
\underset{k}{\forall} \underset{\alpha}{\forall}\left\{\left(k \varepsilon K^{r} \text { y } \alpha P_{k}\right) \Longrightarrow\left[(\alpha, x) \varepsilon E \Longrightarrow x \varepsilon F_{i}\right]\right\}
$$

Esta expresión indica que: Para todos los actos de observación $k$ en el espacio social y para todos los actos de observación $\alpha$ en $x$, si $k$ es un elemento de $K^{r}$ y si los actos de observación $k$ en el espacio social se realizan conjunta pero independientemente de los actos de obsrvación $\alpha$ en $x$, entonces $x$ pertenece a la relación $F_{i}$, si los actos de observación $\alpha$ en $x$ se realizan de acuerdo con la regla $E$.

Entonces, ¿Cuáles son las implicaciones de las proposiciones del tipo 2 para la teorización y la verificación económica?

a) Si los valores observados de $x$ satisfacen $F_{i}$ entonces el consecuente de 2 (es decir la $q$ en la tabla de verdad) ${ }^{30}$ queda confirmado. Pero como puede verse en la tabla, si el consecuente es verdadero, toda la proposición es verdadera, independientemente de que el antecedente sea verdadero o falso. En esta situación toda la proposición 2 queda confirmada;

b) Supóngase ahora que los valores de $x$ no confirman el consecuente de 2 , es decir, los valores observados de $x$ no satisfacen $F_{i}$. En tal caso, según puede verse en la tabla, la verdad de toda la proposición depende del valor de verdad del antecedente. Sin embargo, como los términos del antecedente no han sido interpretados, no es posible afirmar si toda la proposición es o no verdadera. Por lo tanto, las proposiciones del tipo 2 solamente son susceptibles de confirmación por referencia a datos empíricos (esto es, encontrando valores de $x$ de modo de confirmar el consecuente). Mientras el antecedente permanezca sin especificarse, las proposiciones del tipo 2 son solamente confirmables por este camino. Por otra parte, no es posible rechazar afirmaciones como 2, ya que para hacerlo sería necesario saber si la proposición completa es falsa o verdadera; pero esto no puede saberse, a menos que sepamos si el antecedente es falso o verdadero, esto tampoco lo sabemos porque el antecedente no ha sido especificado y por lo tanto no podemos rechazar la proposición completa.

Los sistemas deductivos susceptibles de confirmación pero no de rechazo serán llamados modelos. Si se construye una teoría y se le da la forma de 2, debe especificarse el antecedente, de modo de hacerla susceptible de ser rechazada. Como se vio, esto no puede hacerse con proposiciones del tipo de 2 si el antecedente no ha sido especificado, en cuyo caso la proposición puede confirmarse pero no rechazarse. Los economistas usualmente construyen modelos en lugar de teorías. Construyen sistemas que son confirmables pero no rechazables. ${ }^{31}$

30 La tabla de verdad relevante es la siguiente:

\begin{tabular}{|lll|}
\hline$p$ & $q$ & $p$
\end{tabular}

donde $V$ indica que la proposición tiene un valor verdadero y $F$ un valor falso.

31 Un modelo y una teoría tienen la misma estructura formal pero se diferencian, en nuestro esquema, en cuanto al criterio de verificación a que son suscep- 
Como se dijo antes, Papandreou no trata explícitamente el problema del realismo de los supuestos de las teorías; sin embargo, su análisis ayuda en esta cuestión. La relevancia de los supuestos realistas (es decir, verdaderos) en la economía, depende en mayor medida en si interesa o no rechazar o confirmar sistemas del tipo 2 .

Al economista, como constructor de modelos, le interesa confirmar sistemas del tipo 2, y por lo tanto debe adoptar el enfoque instrumentalista. Le interesa hacer verdadero el consecuente de su sistema deductivo a través de investigación empírica. Dado que al hacer esto no necesita conocer el valor de verdad del antecedente, entonces no tendrá mayor interés por su realismo.

Como constructor de teorías, la tarea del economista es construir sistemas que puedan rechazarse. Y para poder rechazar aquellas teorías inadecuadas (es decir, falsas), el economista debe ser realista. Puesto que para rechazar una teoría es necesario conocer el valor de verdad del antecedente de su sistema deductivo (es decir, que sea un antecedente verdadero), se interesará por tener supuestos realistas en su teoría. Como se dijo antes, solamente cuando el antecedente de una teoria que toma la forma de una proposición condicional es verdadero y su consecuente es falso, entonces podemos rechazarla.

En resumen, el economista deberá ser realista o instrumentalista según sus objetivos. Esta elección dependerá de razones pragmáticas, es decir, de si se interesa por confirmar o bien por refutar proposiciones del tipo 2.

\section{EL PROBLEMA DE LA VERIFICACIÓN EN ECONOMÍA}

Se ha visto que los economistas construyen usualmente sistemas confirmables pero no rechazables, es decir, construyen modelos, no teorías. Se ha visto también que el economista que construye modelos debe hacer verdadero el consecuente de sus sistemas deductivos a través de la investigación empírica, y como constructor de teorías debe hacer verdadero el antecedente.

Sin embargo, el tipo de los supuestos y cláusulas usadas en la investigación empírica no permite la verificación (confirmación o rechazo) de los modelos o teorías. Si estos argumentos son correctos, surgen dudas sobre la posibilidad de construir teorías económicas.

Como ejemplo de verificación de una ley económica, ${ }^{32}$ tomemos la ley de la demanda. Para derivarla, se requieren los supuestos siguientes:

1) Mensurabilidad de la utilidad. En la teoría moderna de la conducta del consumidor no se supone que el consumidor individual posee una medida cardinal de la utilidad derivada del consumo de bienes. Esto es, no se supone que el individuo puede asignar a cada bien un número que representa el monto o grado de utilidad asociado a dicho bien. En vez de ello, se supone simplemente que el consumidor posee una medida

tibles: el modelo puede ser o no confirmado pero no rechazarse. Mientras que una teoría sí puede serlo.

32 Entendemos por ley una proposición de carácter general que toma la forma: de un condicional y que es elemento de una de las clases de proposiciones que constituyen una teoría. 
ordinal de utilidad. Esto significa simplemente que el consumidor puede acomodar los bienes en orden de preferencias.

2) El postulado de racionalidad. El postulado de racionalidad establece que si el consumidor obtiene más de la alternativa $A$ que de la $B$, prefiere $A$ a $B$. Una manera equivalente de formular este postulado es: a) para todas las parejas de alternativas $A$ y $B$, el consumidor sabe si prefiere $A$ a $B \circ B$ a $A$, o si le es indiferente entre una y otra, $b$ ) solamente una de las tres posibilidades es verdadera para cada pareja de alternativas y, c) si el consumidor prefiere $A$ a $B$ y $B$ a $C$, preferirá $A$ a $C$ (esto significa que las preferencias del consumidor son transitivas).

Éstos son dos supuestos de gran importancia en la teoría de la conducta del consumidor en la cual se basa la ley de la demanda. Se hacen luego más supuestos al establecer y verificar dicha ley, pero éstos serán indicados posteriormente.

La ordenación de los bienes que el consumidor hace puede expresarse matemáticamente mediante una "función utilidad" del consumidor. Considérese el caso simple en que el consumidor compra solamente dos artículos.

Su función utilidad queda entonces expresada como:

$$
U=f\left(q_{1}, q_{2}\right)
$$

donde $q_{1}$ y $q_{2}$ son las cantidades de los dos bienes consumidos. Esta función utilidad se define con referencia al consumo durante un intervalo de tiempo.

El consumidor racional desea comprar la combinación de $q_{1}$ y $q_{2}$ de la cual derive el mayor nivel de satisfacción. Su problema es de maximización de la función utilidad. Sin embargo, el ingreso del consumidor es limitado y por ello no puede comprar cantidades ilimitadas de estos i bienes. La restricción presupuestal del consumidor puede escribirse

$$
y_{o}=p_{1} q_{1}+p_{2} q_{2}
$$

donde $y_{o}$ es el ingreso (fijo) y $p_{1}$ y $p_{2}$ son los precios de $q_{1}$ y $q_{2}$ respectivamente. La cantidad gastada en el primer bien $\left(p_{1} q_{1}\right)$ más la cantidad gastada en el segundo $\left(p_{2} q_{2}\right)$ suman un total igual al ingreso $\left(y_{o}\right)$.

El problema del consumidor puede formularse como uno de maximización de la utilidad, sujeto a la restricción presupuestal. Para maximizar la función utilidad sujeta a esta restricción, el consurnidor debe encontrar la combinación de bienes que satisfacen la ecuación presupuestal y que a la vez maximice su función de utilidad.

Las curvas de demanda se derivan del análisis de esta maximización. Considérese el ejemplo siguiente: Supóngase que la función utilidad de un consumidor es

$$
U=q_{1} \cdot q_{2}
$$

y la restricción presupuestal es la expresión 4. Puede entonces formarse la expresión siguiente:

$$
V=q_{1} \cdot q_{2}-\lambda\left(y_{\circ}-p_{1} q_{1}-p_{2} q_{2}\right)
$$


en la cual $\lambda$ es un multiplicador de Lagrange. Para maximizar la función $V$, se obtienen las derivadas parciales de $V$ respecto de las tres variables de la expresión, y tales derivadas se igualan a cero.

$$
\begin{aligned}
& \frac{\partial V}{\partial q_{1}}=q_{2}-p_{1} \lambda=o \\
& \frac{\partial V}{\partial q_{2}}=q_{1}-p_{2} \lambda=o \\
& \frac{\partial V}{\partial \lambda}=y_{o}-p_{1} q_{1}-p_{2} q_{2}=o
\end{aligned}
$$

Las curvas de demanda se obtienen resolviendo este sistema. La solución para $q_{1}$ y $q_{2}$ en término de los parámetros $p_{1}, p_{2}$ y $y_{o}$ resultan:

$$
q_{1}=\frac{y_{o}}{2 p_{1}} \quad, \quad q_{2}:=\frac{y_{o}}{2 p_{2}}
$$

Éstas son las funciones de demanda del consumidor. Dados el ingreso del consumidor y los precios de los bienes, las cantidades demandadas pueden determinarse mediante estas funciones de demanda.

La forma general de la curva de demanda del bien $q_{1}$ puede escribirse/

$$
q_{1}=h\left(p_{1}, p_{2}, y_{o}\right)
$$

o bien, suponiendo que $p_{2}$ y $y_{0}$ son parámetros dados se tiene

$$
q_{1}=g\left(p_{1}\right)
$$

La forma de la función de demanda depende de las propiedades de la función utilidad del consumidor. En general, las curvas de demanda resultan con pendiente negativa: a menor precio mayor cantidad demandada, y viceversa. Ésta es la ley de la demanda.

La ley establece que, ceteris paribus (todas las otras variables permanecen constantes), los consumidores comprarán más de un bien cuando su precio baja. Entre las variables incluidas en la afirmación ceteris paribus se encuentra que los gustos de los consumidores, la disponibilidad de bienes complementarios y sustitutos, deben permanecer constantes y que los mercados deben ser perfectos. La inclusión de esta cláusula tiene implicaciones importantes para la verificación de la ley de la demanda, pero esto se discutirá posteriormente.

La ecuación 8 es una generalización cuantitativa que establece que cuando el precio de un bien $\left(q_{1}\right)$ aumenta la demanda de éste disminuye en $\frac{y_{o}}{2 p_{1}}$. En este caso se tiene una ley cuantitativa.

Nuestro problema ahora es mostrar cómo se verifica la ley de la demanda en la economía. Se acepta generalmente que la verificación de una ley requiere la predicción de un evento y que la observación de los sucesos reales acontezca en una región del tiempo y el espacio específica. 
Considérese por ejemplo, la pređiicción (explicación) ${ }^{33}$ de que aumentará el consumo de un bien determinado. El conjunto $C$ de condiciones iniciales contiene, por ejemplo, la observación de que el precio del bien ha disminuido en cierta cantidad; de que no han cambiado los precios de los sucedáneos conocidos ni de los bienes complementarios y que el ingreso del consumidor tampoco ha cambiado. El conjunto $L$ contiene la ley de la demanda. El conjunto $E$ es el evento económico a ser explicado o pronosticado.

Los economistas consideran que al usar este esquema explicativo, la predicción correcta del conjunto de eventos $E$ (un aumento en el consumo) constituye una confirmación del conjunto de leyes $L$. Este argumento se desarrolla más adelante.

En economía, la verificación de leyes -como la de demanda, que se vale de esquemas explicativos - como la descrita antes, no resulta apropiada por dos razones: a) el carácter único y abierto de los sucesos económicos $\mathrm{y}, b$ ) la cláusula ceteris paribus incluida en las leyes.

\section{El carácter único y abierto de los eventos económicos}

Los sucesos considerados en las ciencias sociales, y en particular en economía, son únicos. Por suceso se entiende todo lo que acontece en una región del tiempo y el espacio y por único el carácter no repetitivo, es decir, histórico de los sucesos.

Para explicar el carácter abierto de los sucesos económicos, y dado que esta característica se relaciona con la verificación de las leyes económicas, se hace primero un repaso sobre la forma en que se relacionan la explicación de los sucesos y su verificación en las ciencias físicas.

La explicación ${ }^{34}$ de un suceso o una clase de sucesos consiste en un conjunto $E$ de observaciones que definen el explicandum; un conjunto de leyes generales $L$, y un conjunto $C$ de observaciones antecedentes que describen el explicando. $C$ está relacionado con $E$ mediante las leyes generales en $L$.

Un sistema cerrado es aquel en el cual los conjuntos $E$ y $C$ son finitos y exhaustivos. La explicación del evento único en un sistema no cetrado implica, por otra parte, que $E$ y $C$ son infinitamente grandes. El profesor Hempel introduce el concepto de esbozo de explicación ${ }^{35}$ para denotar la explicación incompleta del sucéso único. El proceso de mejoramiento de este cuadro explicativo mediante la inclusión de más y más entidades observables puede continuarse indefinidamente, hasta que el proceso es interrumpido por la decisión del investigador. Esta idea de ésbozo de explicación se utilizará para explicar los sucesos únicos.

Se dará ahora un ejemplo de cómo se utilizan en las ciencias físicas estos conceptos de explicación y de esbozo de explicación.

Considérese primero el caso de la explicación de un suceso en un sistema cerrado. Tómese el caso de un monobloc (la caja del motor)

33 Se sigue aquí el argumento de Hempel que interpreta las diferencias entre explicar y predecir como progmáticas. Véase "The Logic of Explanation" en C. G. Hempel, Readings in the Philosophy of Science, H. Feigl y May Brodbeck, Nueva York, 1953, p. 322.

34 Hempel, ibid.

35 "The Function of General Laws in History" en H. Feigl y W.: Sellors (Comp.), Readings in Philosophical Analysis, Nueva York, 1949. 
agrietado que se encuentra en un laboratorio bajo condiciones controladas. El conjunto $E$ incluye las observaciones siguientes: existe un monobloc; está hecho de acero; tiene una grieta; el agua del sistema de enfriamiento está a una temperatura de $100^{\circ}$ centígrados. El conjunto $C$ incluye: que la temperatura del laboratorio durante las últimas 10 horas estuvo por debajo de $0^{\circ} \mathrm{C}$; y que el agua no contiene preparaciones anticongelantes. El conjunto $L$ contiene leyes específicas acerca de la conducta del agua a varias temperaturas y acerca del efecto de expansión del hielo en las paredes de acero del monobloc. La explicación consiste entonces en que, durante las diez horas precedentes, el agua del sistema de enfriamiento se congeló y que la expansión resultante ocasionó la fractura del monobloc. En $E$ se han incluido solamente las observaciones consideradas pertinentes. Por consiguiente, si se encontrara intacto el monobloc y todas las condiciones permanecieran iguales, se consideraría que al menos una de las leyes de $L$ no ha sido confirmada. En este caso la explicación de un suceso en un sistema cerrado implica la verificación de las leyes utilizadas en el esquema explicativo.

Considérese ahora el esbozo de explicación de un suceso en un sistema abierto. Supóngase que encontramos que el monobloc de un automóvil que ha estado a la intemperie durante varios días está agrietado. Supóngase además que no se dispone de informes confiables sobre la temperatura durante las últimas 24 horas, y que el agua se ha filtrado del sistema de enfriamiento, de modo que no puede establecerse su composición química. La explicación en este caso se hace en términos de las mismas leyes y de las mismas entidades observables, igual que en el caso del sistema cerrado, pero la explicación introduce ahora, hipotéticamente, las observaciones faltantes de las variables. Por ejemplo, que la temperatura en algún momento debió haber sido inferior a $0^{\circ} \mathrm{C}$ y que el sistemá de enfriamiento pudo no haber tenido la cantidad suficiente de anticongelante. En este ejemplo las leyes del conjunto $L$ no se verifican mediante el hallazgo del monobloc agrietado. Por el contrario, precisamente porque estas leyes han sido verificadas y aceptadas previamente, es posible deducir en el esbozo de explicación las condiciones que pudieron haber ocasionado la fractura del monobloc.

De este modo, la identidad entre explicación y verificación es válida solamente en un sistema cerrado. El esbozo de explicación de un suceso en un sistema abierto requiere el uso de leyes previamente verificadas y aceptadas. Estas leyes deben ser verificadas independientemente de su aplicación a la explicación de un suceso en un sistema abierto.

Respecto de la explicación y la verificación de los sucesos económicos y la verificación de las leyes económicas, conviene hacer las consideraciones siguientes:

1) Los sucesos explicados por la economía corresponden a la clase der sucesos que acontecen en un sistema abierto. La explicación de estos sucesos no es completa, sino un esbozo de explicación en el cual no es válido identificar explicación con verificación. Sin embargo, se encuentra que en economía los estudios econométricos efectuados para verificar las leyes, trátan las explicaciones de los sucesos económicos como explicaciones completas, y las consideran luego como la 
verificación de las leyes. Este tratamiento es erróneo, ya que la identidad mencionada no es válida en sistemas abiertos.

2) Además, en economía se encuentra otra complicación que está ausente en la explicación de los sucesos de sistemas abiertos del tipo del ejemplo del monobloc agrietado. Como se vio, la explicación de un suceso en un sistema abierto (el monobloc a la intemperie) requiere de leyes verificadas previamente. Estas leyes deberán verificarse independientemente de su utilización en el esbozo de explicación. Así, leyes referentes al comportamiento del agua a diferentes temperaturas y la resistencia del hierro a la presión, pueden verificarse previa $\mathrm{e}$ independientemente de su utilización en el esbozo de explicación del monobloc agrietado dejado a la intemperie. Más aún, puede decirse que estas leyes se usan en este esbozo de explicación porque ya han sido verificadas y aceptadas.

Por otra parte, puesto que los sucesos económicos acontecen en sistemas abiertos, su explicación requiere el uso de leyes verificadas previamente. Esta verificación previa no puede hacerse en la ciencia económica. Las leyes económicas no pueden ser verificadas independientemente de su uso en el esbozo de explicación, ya que todos los casos en que se aplican son históricos, es decir, sucesos únicos que ocurren en sistemas abiertos. (Véase antes la especificación temporal dada a la función utilidad al derivar la ley de la demanda). En otras palabras, los economistas no pueden realizar experimentos de laboratorio para verificar las leyes económicas previamente a su uso en la explicación de un suceso.

Para resumir, en economía no es posible verificar una ley económica mediante la explicación de un suceso ni acudir a leyes económicas cuando se utiliza un esbozo de explicación de un suceso dado, ya que esto requiere de la verificación previa de tales leyes, lo cual no es posible en la ciencia económica.

Lo que los economistas hacen equivocadamente para salvar el obstáculo es formular leyes económicas sujetas a la cláusula ceteris paribus y tratar los sucesos económicos como parte de un sistema cerrado.

\section{La cláusula ceteris paribus}

Al verificar las leyes económicas, los economistas usualmente tratan los sucesos como parte de un sistema cerrado, práctica que hemos visto es errónea y formulan las leyes económicas sujetas a la cláusula de ceteris paribus. Este enfoque hace que las leyes sean inverificables.

Considérese el ejemplo de explicar o predecir un aumento en la demanda de un bien determinado. Si se hacen predicciones correctas, se considera que la ley de la demanda queda verificada. Por otra parte, si el consumo no aumenta no se considera que la ley de demanda quede refutada. Sin embargo, en lugar de decir que se ha hecho un juicio equivocado, los economistas acuden al expediente de decir que un descenso en los precios "tiende a..." o de introducir la frase ceteris paribus. Esto no deja lugar a la verificación de las leyes, ya que en el primer caso siempre puede exculparse a la ley diciendo "lo único que se intentaba era mostrar una tendencia y deben haber existido tendencias opuestas": 
y en el segundo caso puede exculparse la ley diciendo "cambió algo que se suponía constante". En otras palabras, el hecho de que el valor prot nosticado de una o más variables no se observe en la región del tiempo y el espacio especificados, no conduce al rechazo de las leyes utilizadas para hacer la predicción; es siempre posible explicar la discrepancia entre los sucesos observados y pronosticados acudiendo al argumento de que la condición ceteris paribus no se cumplió. El inverso del razonamiento usual está presente: el descubrimiento de que la ley no predice acertadamente, sirve como premisa de la cual se deriva la conclusión de que una o más de las variables cubiertas bajo la condición de ceteris paribus debió haber cambiado.

Las leyes de otras ciencias frecuentemente tienen la cláusula ceteris paribus, a menudo implícitamente, pero sí es posible especificar con exactitud lo que está incluido dentro de las "otras cosas". Sin embargo, en-la economía la cláusula confunde la cuestión. O bien no se especifican las variables incluidas en la cláusula de modo que el supuesto de algún cambio en el mundo económico salva la ley, o bien las variables que aparecen en la lista, tales como los gustos del consumidor, los ingresos reales, etc., son aquellas cuyo cambio es extremadamente difícil de medir, lo cual hace conveniente introducirlas en la cláusula (ver antes los supuestos alrededor de las leyes de la demanda).

En resumen, se mencionan los tres problemas a los que se enfrentany los economistas al tratar de verificar las leyes económicas. Primero, los economistas no pueden verificar tales leyes mediante la explicación de los sucesos económicos, ya que éstos acontecen en sistemas abiertos donde la explicación del suceso y la verificación de las leyes no pueden identificarse. Segundo, ya que los sucesos económicos son históricos y acontecen en sistemas abiertos, en su explicación (esbozo de explicación) no puede acudirse a leyes económicas. Las leyes utilizadas en el esbozo de explicación de un suceso, en un sistema abierto, deben ser verificadas previamente a su uso, pero esto no puede hacerse porque los sucesos económicos son únicos. Tercero, para eludir el problema los economistas no solamente tratan los sucesos como si ocurrieran en sistemas cerrados, sino que insertan la cláusula ceteris paribus que hace inverificables las leyes. 